\title{
Characterization of Super Strongly Perfect Graphs in Chordal and Strongly Chordal Graphs
}

R Mary Jeya Jothi* and A Amutha ${ }^{\dagger}$

\begin{abstract}
A Graph G is Super Strongly Perfect Graph if every induced sub graph $H$ of $G$ possesses a minimal dominating set that meets all the maximal complete sub graphs of $\mathrm{H}$. In this paper, we have investigated the characterization of Super Strongly Perfect graphs using odd cycles. We have given the characterization of Super Strongly Perfect graphs in chordal and strongly chordal graphs. We have presented the results of Chordal graphs in terms of domination and co - domination numbers $\gamma$ and $\bar{\gamma}$. We have given the relationship between diameter, domination and co - domination numbers of chordal graphs. Also we have analysed the structure of Super Strongly Perfect Graph in Chordal graphs and Strongly Chordal graphs.
\end{abstract}

Keywords: Super strongly perfect graph, minimal dominating set, domination and co-domination numbers and chordal graphs.

* Research Scholar, Department of Mathematics, Sathyabama University, Chennai, Tamil Nadu. jeyajothi31@gmail.com

† Assistant Professor, Department of Mathematics, Sathyabama University, Chennai, Tamil Nadu. 


\section{Introduction}

In Mathematics, a graph is an abstract representation of a set of objects where some pairs of the objects are connected by links. The interconnected objects are represented by mathematical abstractions called vertices, and the links that connect some pairs of vertices are called edges. Graphs are one of the objects of study in discrete mathematics. Graphs in this context differ from the more familiar co - ordinate plots that portray mathematical relations and functions [3]. Although graph theory is one of the younger branches of mathematics, it is fundamental to a number of applied fields, including operations research, computer science, and social network analysis. Graph theory has proven useful in the design of integrated circuits for computers and other electronic devices. These components, more often called chips, contain complex, layered microcircuits that can be represented as sets of points interconnected by lines or arcs [8]. Using graph theory, engineers develop chips with maximum component density and minimum total interconnecting conductor length. This is important for optimizing processing speed and electrical efficiency [4].

The chordal graphs have been intensively studied in 1960's. The class of chordal graphs is a class of crucial importance. They represent an example of a class that is a generalization of trees with many different characterizations, rich structural properties, and many important applications [11]. These graphs appear under other names such as triangulated graphs, rigid - circuit graphs, monotone transitive graphs, maximal planar graphs and perfect elimination graphs in the literature [1]. Reconstructing the histories of language families is one of the principal tasks of historical linguistics. A linguistic phylogenetic tree conveys the evolution of a language family. The family tree can be constructed on the basis of characteristics that are common to sets of languages which include lexical, phonological, and morphological affinities. Jessica and Grzegorz proposed a heuristic search algorithm based on the notion of chordal graphs to inferring phylogenetic trees of languages [12]. 


\section{Preliminaries}

In this paper, graphs are finite and simple, that is, they have no loops or multiple edges. Let $\mathrm{G}=(\mathrm{V}, \mathrm{E})$ be a graph. The complement of a graph $G$ denoted by $\bar{G}$, is a graph $H$ on the same vertices such that two vertices of $\mathrm{H}$ are adjacent if and only if they are not adjacent in $\mathrm{G}$. A clique in $\mathrm{G}$ is a set $\mathrm{X} \subseteq \mathrm{V}(\mathrm{G})$ of pair wise adjacent vertices. A subset $D$ of $V(G)$ is called a dominating set if every vertex in $\mathrm{V}-\mathrm{D}$ is adjacent to at least one vertex in $\mathrm{D}$. A subset $\mathrm{S}$ of $\mathrm{V}$ is said to be a minimal dominating set if $\mathrm{S}-\{\mathrm{u}\}$ is not a dominating set for any $u \in S$. The domination number $\gamma(G)$ of $G$ is the smallest size of a dominating set of $G$. The domination number of its complement $\bar{G}$ is called the co - domination number of $G$ and is denoted by $\gamma(\bar{G})$ or simply $\bar{\gamma}$. A shortest uv path of a connected graph $\mathrm{G}$ is often called a geodesic. The diameter denoted by diam $(G)$ is the length of any longest geodesic. The graph is perfect, if in all its induced sub graphs, the size of the largest clique is equal to the chromatic number. A simplicial vertex in a graph is a vertex whose neighbours form a clique. A simple vertex in a graph is a vertex such that the closed neighbourhoods of its neighbours form a chain under inclusion. (The closed neighbourhood $\mathrm{N}[\mathrm{v}]$ of a vertex $v$ is $N(v) \cup v$ where $N(v)=\{u: u v \in E(G)\}$ Note that a simple vertex is also simplicial.

\section{Our Results on Super Strongly Perfect Graph}

In this paper, we have given the characterization of Super Strongly Perfect graphs using odd cycles. Using this characterization we have characterized the Super Strongly Perfect graphs in Chordal graphs and Strongly chordal graphs. We have presented the results of Chordal graphs in terms of domination and co - domination numbers $\gamma$ and $\bar{\gamma}$. Also we have discussed the structure of Super Strongly Perfect Graph in Chordal graphs and Strongly Chordal graphs. 


\subsection{Super Strongly Perfect Graph}

A Graph $G=(V, E)$ is Super Strongly Perfect if every induced sub graph $H$ of $G$ possesses a minimal dominating set that meets all the maximal complete sub graphs of $\mathrm{H}$.

\section{Example 1}

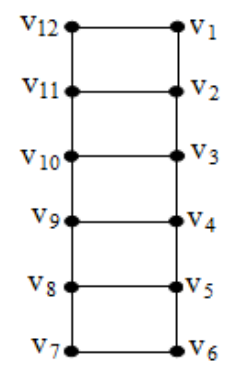

Figure 1: Super Strongly Perfect Graph

Here, $\left\{v_{1}, v_{3}, v_{5}, v_{7}, v_{9}, v_{11}\right\}$ is a minimal dominating set which meet all maximal cliques of $\mathrm{G}$.

\section{Example 2}

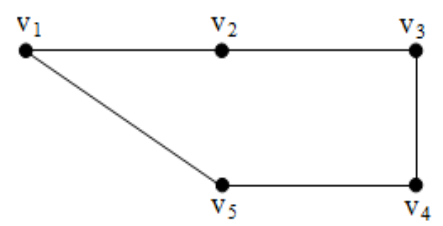

Figure 2: Non - Super Strongly Perfect Graph

Here, $\left\{\mathrm{v}_{1}, \mathrm{v}_{3}\right\}$ is a minimal dominating set which does not meet all maximal cliques of $G$.

\section{Cycle Graph}

A cycle is a path (a path in a graph is a sequence of vertices such that from each of its vertices there is an edge to the next vertex in the sequence) such that the start vertex and end vertex are the same and it is denoted by $C_{n}$. The number of vertices in $C_{n}$ equals the number of edges. The cycle graph with even number of vertices is called an even cycle the cycle graph with odd number of vertices is called an odd cycle. 


\section{1. Theorem [2]}

Let $G$ be a graph. Then G is Super Strongly Perfect if and only if it does not contain an odd cycle, $n \geq 5$ as an induced sub graph.

\section{Chordal Graph}

A graph $G$ is chordal if each cycle in $G$ of length at least four has at least one chord. The class of chordal graphs is one of the first class which has been studied at the beginning of the theory of perfect graphs, and it has several characterizations [11]. The chordal graphs are a subclass of the well known perfect graphs. Other superclasses of chordal graphs include the weakly chordal graphs, the odd - hole - free graphs, and the even - hole - free graphs [5]. A graph $G$ is chordal if and only if it has perfect elimination property [4]. An important one due to Gavril [9], characterizes the class of chordal graphs as the intersection graphs of a family of sub trees of a tree. Dirac [6] proved that a graph is chordal if and only if every induced subgraph has a simplicial vertex.

\section{Example}

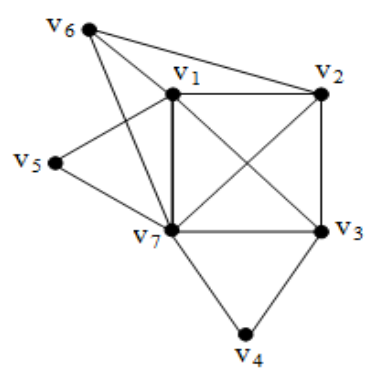

Figure 3: Chordal Graph

Here, $\left\{\mathrm{v}_{4}, \mathrm{v}_{7}\right\}$ is a minimal dominating set which meets all maximal cliques of $\mathrm{G}$.

\subsection{Theorem}

Every Chordal graph is Super Strongly Perfect. 


\section{Proof:}

Let $\mathrm{G}$ be a Chordal graph.

$\Rightarrow G$ does not contain an odd cycle as an induced sub graph.

Now, by the theorem 4.1, G is Super Strongly Perfect.

Hence every Chordal is Super Strongly Perfect.

\subsection{Theorem}

Let $G$ be a Chordal graph which is Super Strongly Perfect, then $\operatorname{diam}(G)=\gamma(G)=1$ if and only if $G$ is complete.

\section{Proof:}

Let $\mathrm{G}$ be a Chordal graph which is Super Strongly Perfect.

Assume diam $(\mathrm{G})=\gamma(\mathrm{G})=1$.

To prove $\mathrm{G}$ is complete.

Suppose $\mathrm{G}$ is not complete.

Then there exists at least two non adjacent vertices say $u, v$ in $G$ such that diam $(\mathrm{u}, \mathrm{v})>1$.

$\Rightarrow \operatorname{diam}(\mathrm{G})>1$ also $\gamma(\mathrm{G})>1$.

This is a contradiction to the assumption.

Hence $\mathrm{G}$ is complete.

Conversely assume that $\mathrm{G}$ is complete.

To prove $\operatorname{diam}(\mathrm{G})=\gamma(\mathrm{G})=1$.

Suppose $\operatorname{diam}(\mathrm{G}) \neq 1$ and $\gamma(\mathrm{G}) \neq 1$.

Case 1: $\operatorname{diam}(\mathrm{G}) \neq 1$.

Then there exists at least two vertices say $u, v$ in $G$ such that $\operatorname{diam}(\mathrm{u}, \mathrm{v})>1$.

$\Rightarrow \mathrm{u}$ and $\mathrm{v}$ are non adjacent in G. This is a contradiction to the assumption. Hence diam $(\mathrm{G})=1$. 
Case 2: $\quad \gamma(G) \neq 1$.

Then there exists at least one vertex which is not adjacent to some vertices in $\mathrm{G}$.

This is a contradiction to the assumption.

Hence $\gamma(G)=1$.

\subsection{Theorem}

Let $G$ be a Chordal graph which is Super Strongly Perfect, then $\gamma(\bar{G}) \geq 3$ if and only if $\operatorname{diam}(G) \leq 2$.

\section{Proof:}

Let $\mathrm{G}$ be a Chordal graph which is Super Strongly Perfect.

Assume $\gamma(\overline{\mathrm{G}}) \geq 3$.

To prove $\operatorname{diam}(\mathrm{G}) \leq 2$.

Suppose diam $(\mathrm{G})>2$.

Then there exists at least two vertices say $u, v$ in $G$ such that $\operatorname{diam}(\mathrm{u}, \mathrm{v}) \geq 3$.

Then there does not exist a vertex in $\bar{G}$ which is adjacent to both $u$ and $\mathrm{v}$.

$\Rightarrow$ All the vertices in $\bar{G}$ are either adjacent to $u$ or $v$.

$\Rightarrow \gamma(\overline{\mathrm{G}}) \leq 2$.

This is a contradiction to the assumption.

Hence diam $(G) \leq 2$.

Conversely assume that $\operatorname{diam}(\mathrm{G}) \leq 2$.

To prove $\gamma(\overline{\mathrm{G}}) \geq 3$.

Suppose $\gamma(\overline{\mathrm{G}}) \leq 2$.

Then there exists a minimum dominating set say, $D=\{u, v\}$ for some $u, v$ in $\bar{G}$.

$\Rightarrow$ All the vertices in $\bar{G}$ are either adjacent to $u$ or $v$. 
Then there does not exist a vertex in $G$ which is adjacent to both $u$ and $v$.

$\Rightarrow \operatorname{diam}(\mathrm{u}, \mathrm{v}) \geq 3$.

$\Rightarrow \operatorname{diam}(\mathrm{G}) \geq 3$.

This is a contradiction to the assumption.

Hence $\gamma(\overline{\mathrm{G}}) \geq 3$.

\subsection{Theorem}

Let $G$ be a Chordal graph with isolated vertices which is Super Strongly Perfect, then $\gamma(\overline{\mathrm{G}})=1$ if and only if diam $(\mathrm{G})$ is undefined.

\section{Proof:}

Let $G$ be a Chordal graph with isolated vertices which is Super Strongly Perfect.

Assume $\gamma(\overline{\mathrm{G}})=1$.

To prove diam $(\mathrm{G})$ is undefined.

Since $\gamma(\overline{\mathrm{G}})=1$, there exists at least one vertex say $\mathrm{u}$ in $\overline{\mathrm{G}}$ which is adjacent to all the remaining vertices in $\overline{\mathrm{G}}$.

$\Rightarrow \mathrm{u}$ is an isolated vertex in $\mathrm{G}$.

$\Rightarrow$ diam $(\mathrm{G})$ is undefined.

Conversely assume that diam $(\mathrm{G})$ is undefined.

Then there exists at least one isolated vertex say $\mathrm{u}$ in $\mathrm{G}$.

$\Rightarrow \mathrm{u}$ is adjacent to all the remaining vertices in $\overline{\mathrm{G}}$.

$\Rightarrow \gamma(\overline{\mathrm{G}})=1$.

\subsection{Proposition}

Let $G$ be a Chordal graph with no isolated vertices which is Super Strongly Perfect, then diam $(G) \geq 3$ if and only if $Y(\bar{G})=2$. 


\subsection{Proposition}

Let $G$ be a Chordal graph with $\gamma(G)=\gamma(\bar{G})$ which is Super Strongly Perfect, then $Y(G)=\gamma(\bar{G}) \geq 3$ if and only if $2 \leq \operatorname{diam}(\overline{\mathrm{G}}) \leq 5$.

\section{Strongly Chordal Graph}

A chordal graph is said to be strongly chordal if every cycle on six or more vertices contains a chord joining two vertices with an odd distance between them. The class of strongly chordal graphs is an interesting subclass of chordal graphs. Many combinatorial graph problems which are NP - complete in chordal graphs are polynomially solvable in strongly chordal graphs [10]. The analogue by Farber [7] is that a graph is strongly chordal if and only if every induced sub graph has a simple vertex. Strongly chordal graphs may also be characterized as the graphs having a strong perfect elimination ordering, an ordering of the vertices such that the neighbours of any vertex that come later in the ordering form a clique and such that, for each $i<j<k<l$, if the $i^{\text {th }}$ vertex in the ordering is adjacent to the $k^{\text {th }}$ and the $l^{\text {th }}$ vertices, and the $j^{\text {th }}$ and $k^{\text {th }}$ vertices are adjacent, then the $j^{\text {th }}$ and $l^{\text {th }}$ vertices must also be adjacent [7].

\section{Example}

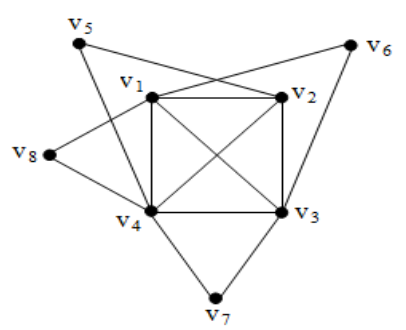

Figure 4: Strongly Chordal Graph

Here, $\left\{\mathrm{v}_{3}, \mathrm{v}_{4}\right\}$ is a minimal dominating set which meet all maximal cliques of $\mathrm{G}$. 


\subsection{Theorem}

Every Strongly Chordal graph is Super Strongly Perfect.

\section{Proof:}

Let $\mathrm{G}$ be a Strongly Chordal graph.

$\Rightarrow \mathrm{G}$ does not contain an odd cycle as an induced sub graph.

Now, by the theorem 4.1, G is Super Strongly Perfect.

Hence every Strongly Chordal graph is Super Strongly Perfect.

\section{Conclusions}

We have given the characterization of Super Strongly Perfect graphs using odd cycles. We have presented the characterization of Super Strongly Perfect graphs in chordal graphs and strongly chordal graphs. Also we have investigated the structure of Super Strongly Perfect Graph in chordal graphs and strongly chordal graphs. As well we can have the good scope of well known other architectures like Butterfly graphs, Benes Butterfly graphs etc.

\section{References}

[1] A Amutha, "Tree spanners of symmetric interconnection networks", $\quad$ Ph.D. thesis, University of Madras, October 2006.

[2] A Amutha and R M J Jothi, Characterization of super strongly perfect graphs in bipartite graphs, Proceedings of an International Conference on Mathematical Modelling and Scientific Computation, vol. 1, pp. 183-185, 2012.

[3] J A Bondy and U S R Murty, Graph Theory with Applications, Elsevier Science, North Holland, 1976.

[4] A Brandstadt, V B Le and J P Spinrad, Graph Classes: A Survey, SIAM Monographs on Discrete Mathematics and Applications, 1999.

[5] Diestel and Reinhard, Graph Theory, Graduate Texts in Mathematics, Springer-Verlag, vol. 173, pp. 6-9, 2005. 
[6] G A Dirac, On Rigid Circuit Graphs, Abh. Math. Sem. Univ. Hamburg, Springer-Verlag, vol. 25, pp. 71-76, 1961.

[7] M Farber, Characterization of strongly chordal graphs, Discrete Mathematics, vol. 43, pp. 173-189, 1983.

[7] P C Fishburn, Utility Theory and Decision Making, Wiley, New York, 1970.

[8] F Gavril, The intersection graphs of subtrees are exactly the chordal graphs, Journal of Combinatorics Theory B, vol. 16, pp. 47-56, 1974.

[9]A Gibbons, Algorithmic Graph Theory, Cambridge University Press Publications, 1985.

[10] M C Golumbic, Algorithmic Graph Theory and Perfect Graphs, Academic Press, New York, 1980.

[11] J Enright and G Kondrak, The application of chordal graphs to inferring phylogenetic trees of languages", Proceedings of the 5th International Joint Conference on Natural Language Processing, pp. 545552, Chiang Mai, Thailand, November 8 - 13, 2011. 